\title{
Effects of hormone replacement therapy on magnetic resonance imaging of brain parenchyma hyperintensities in postmenopausal women
}

\author{
Yan-yong LIU ${ }^{1}$, Ling HU² ${ }^{2}$, Chao JI ${ }^{1}$, Dong-wen $\mathrm{CHEN}^{1}$, Xi SHEN ${ }^{1}$, Nan YANG ${ }^{1}$, Yun YUE ${ }^{1}$, Jing-mei JIANG ${ }^{1}, \mathrm{Xia} \mathrm{HONG}^{2}$, \\ Qin-sheng $\mathrm{GE}^{2}$, Ping-ping ZUO $\mathrm{Z}^{1, *}$ \\ ${ }^{1}$ Department of Pharmacology, School of Basic Medicine, Peking Union Medical College and Institute of Basic Medical Sciences, \\ Chinese Academy of Medical Sciences, Beijing 100005, China; ${ }^{2}$ Peking Union Medical College Hospital, Beijing 100730, China
}

\begin{abstract}
Aim: To apply 3.0 magnetic resonance imaging (MRI) to study the effects of long-term, low dose hormone replacement therapy (HRT) on the brain parenchyma of postmenopausal women.

Methods: A total of 155 postmenopausal healthy female medical staff members from Peking Union Medical College Hospital were enrolled. The HRT group was composed of 71 subjects who had been given a low dose of HRT for over 4 years, while 84 women who had never been given HRT were enrolled in the control group. The Mini-Mental State Examination (MMSE) was used to evaluate mental state, and an Enzyme-Linked ImmunoSorbent Assay (ELISA) was used to detect plasma levels of sex hormones. In addition, all participants were subjected to an MRI, including axial T2 weighted imaging (T2WI), fluid-attenuated inversion recovery (FLAIR), T1 weighted imaging (T1WI, oblique coronal, vertical to the hippocampus, slice thickness $3 \mathrm{~mm}$ without gaps), and a 3D image of the whole brain. Results: The ELISA showed that the plasma level of estradiol in the HRT group was significantly higher than that in the control group $(P<0.05)$. No differences were observed in the MMSE between the two groups. In participants older than 70 years of age, the number of deep white matter hyperintensities (DWMHs) in the control group was significantly higher than that in the HRT group ( $P=0.0013$ ); however, in other age subgroups, no statistical differences were observed. Finally, no significant difference in periventricular hyperintensity $(\mathrm{PVH})$ between the two groups was observed.

Conclusion: We found that a high plasma level of estradiol in postmenopausal women receiving long-term HRT was correlated with the survival of brain parenchyma.
\end{abstract}

Keywords: postmenopausal women; hormone replacement therapy; estrogen; magnetic resonance imaging; deep white matter hyperintensity

Acta Pharmacologica Sinica (2009) 30: 1065-1070; doi: 10.1038/aps.2009.81

\section{Introduction}

Considerable evidence suggests that in both animals and humans, estrogen used as hormone replacement therapy (HRT) can have positive effects on cognitive performance in aging. There are, however, contradictory opinions regarding HRT. The Women's Health Initiative Memory Study project, completed in 2004, came to the conclusion that estrogen therapy alone did not reduce dementia, but instead increased the risk $^{[1]}$. It should be noted that the main differences between the current and the latter project lie in the administrated dosage (less than recommended dosage $v$ s routine dosage) and the age of HRT initiation in the subjects (mean 54.9 years vs 65

*To whom correspondence should be addressed. E-mail zuopp@126.com

Received 2008-12-19 Accepted 2009-04-29 years). While numerous studies have examined the effects of HRT on cognitive function, few studies have focused on the influence of HRT on brain integrity in aging ${ }^{[2]}$. Eberling et al found that postmenopausal women using HRT had a larger right hippocampus compared with non-users ${ }^{[3]}$. In addition, there is evidence from a longitudinal study of HRT-related sparing of age-related decline in cortical regions ${ }^{[4]}$. Moreover, it has been shown that women using HRT showed a selective sparing of brain tissue decline with aging as a function of treatment duration, with gray matter being preserved in the prefrontal, parietal, and temporal regions and white matter in the medial temporal lobe regions ${ }^{[5]}$. Several volumetric studies, however, have not detected differences between HRT users and non-users in various brain regions, such as the frontal, parietal, prefrontal, and temporal regions (including the hippocampus $)^{[6,7]}$ or in intracranial volume $(\mathrm{ICV})^{[8]}$. One study 
found larger right hippocampal volumes in HRT users, but no differences in whole hippocampal volumes ${ }^{[9]}$. Another study found that previous and current HRT users had more gray matter in the frontal, prefrontal, and temporal regions, more white matter in the medial temporal lobe, and less cerebrospinal fluid overall ${ }^{[5]}$.

In summary, structural magnetic resonance imaging (MRI) studies have presented mixed results, showing larger and smaller brain volumes, and no or negative effects on white matter. Because of these inconsistencies, the effects of HRT on the human brain require further investigation. Using MRI in postmenopausal women, we have reported that changes in hippocampal volume are related to sharp decreases in learning and memory function as age progresses, findings that are consistent with a study showing that hippocampal atrophy in elderly men was significantly related to aging ${ }^{[10]}$. We have also reported that long-term, low dose HRT can slow this trend, especially for women 65 and older ${ }^{[11]}$. Most studies currently adopt the intelligence evaluation table in order to evaluate the effects of HRT on postmenopausal women, while few focus on the transformation of brain parenchyma. In this study, we employ MRI to further evaluate the effects of HRT on deep white matter hyperintensity (DWNH) and periventricular hyperintensity $(\mathrm{PVH})$ in the brains of postmenopausal women.

\section{Materials and methods Study population and groups}

A total of 182 postmenopausal female medical staff members from the Peking Union Medical College Hospital (PUMC, Beijing, China) participated in this study. The HRT group was composed of 83 women with an average age of 66.3 years (range 51-85 years). They had all received HRT (half or one quarter of the dosage) for at least 4 years after menopause initiation. The control group was composed of 99 age-matched women with an average age of 67.1 years (range 51-87 years). The women in the control group had never received HRT. The mean age of menopause onset in the HRT group was 48.7 years, and the mean age of HRT initiation was 54.9 years. All participants had MMSE scores above 26, indicating that they had normal personalities and emotions. A threshold of 26 was chosen according to the MMSE score investigation performed on the Beijing population, which was obtained using the same normalized method ${ }^{[12]}$. None of the participants ever had diseases such as hemiplegia or Alzheimer's disease or a history of other nervous system disorders or symptoms. The subjects were divided into three subgroups according to their age: 50-59, 60-69, and 70-87 years. The study was approved by the institutional review board of the Ethics Committee of $\mathrm{PUMCH}$, and all participants gave written informed consent.

\section{Administrated hormones}

The hormones used included estradiol valerate (Progynova, recommended dosage $1.0 \mathrm{mg} / \mathrm{d}$ ), conjugated equine estrogen (Premarin, $0.3 \mathrm{mg} / \mathrm{d}$ ), tibolone (Livial, $2.5 \mathrm{mg} / \mathrm{d}$ ) and medroxyprogesterone acetate (Progevera, $2.0 \mathrm{mg} / \mathrm{d}$ ). Among the 83 HRT subjects, 9 used half of the recommended dosage,
52 used one quarter of the dosage and 22 used less than one quarter of the dosage.

\section{Global cognitive function evaluation}

We used the Mini-Mental State Examination (MMSE) as a general index of cognition ${ }^{[13]}$. The MMSE is a list of 30 questions and tasks covering orientation to time and place, word registration and recall, attention and calculation, language and visual construction. The MMSE is one of the tests recommended by the National Institute of Neurological Disorders and Stroke and the Alzheimer's Disease and Related Disorders Association to document the diagnosis of Alzheimer's disease ${ }^{[14]}$.

A total of 11 questions from the MMSE represent four intelligence-relating subjects: (1) cognition (orientation to place and time, calculation ability), composed of the score sum of questions 1,2 , and 4 ; (2) memory (retention and recall memory), composed of the score sum of questions 3 and 5; (3) language (naming, retelling, read, comprehension, spontaneous writing), composed of the score sum of questions 6, 7, 9, and 10; and (4) executive function and visuo-constructional abilities (copy graphics), composed of the score sum of questions 8 and 11.

\section{Blood sample preparation and measurements}

A total of $10 \mathrm{~mL}$ of fasting blood was collected from each participant by vein puncture. Samples were placed into prepared tubes containing EDTA- $\mathrm{K}_{2}$ and stored at $4{ }^{\circ} \mathrm{C}$ for $30 \mathrm{~min}$. Subsequently, the blood samples were centrifuged at $4{ }^{\circ} \mathrm{C}, 1000 \times g$ for $15 \mathrm{~min}$ and the plasma were collected for assay. The plasma levels of estradiol $\left(\mathrm{E}_{2}\right)$, progesterone $(\mathrm{P})$ and testosterone $(\mathrm{T})$ were analyzed by Enzyme-Linked ImmunoSorbent Assay (ELISA) (Model 550, Bio-Rad, Hercules, CA, USA). Blood lipids, glucose and pressure were also measured to exclude ordinary risk factors for white matter lesions.

\section{Magnetic resonance imaging}

Magnetic resonance imaging (GE Sigma 3.0T VHIEXITE3, General Electric Medical Systems, Milwaukee, WI, USA) was performed on each subject. The scanning protocol included axial T2 weighted imaging (T2WI), fluid-attenuated inversion recovery (FLAIR), T1 weighted imaging (T1WI, oblique coronal, vertical to the hippocampus, slice thickness $3 \mathrm{~mm}$ without gaps) and a 3D image of the whole brain. DWMH and PVH were chosen as study indices and were evaluated using Fazekas' visual rating scale (Figures 1 and 2). All images were ana-

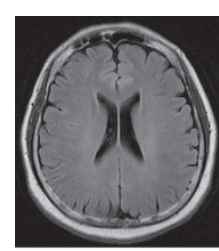

$\mathrm{DWMH}=0$

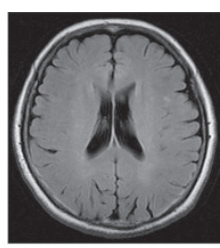

$\mathrm{DWMH}=1$

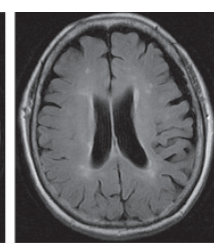

$\mathrm{DWMH}=2$

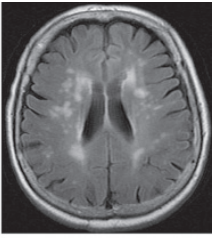

$\mathrm{DWMH}=3$
Figure 1. DWMH imaging grading. 


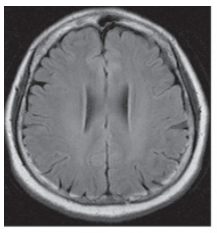

$\mathrm{PVH}=0$

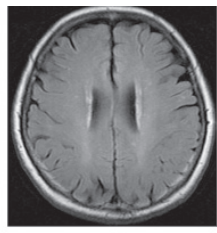

$\mathrm{PVH}=1$

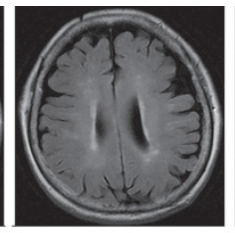

$\mathrm{PVH}=2$

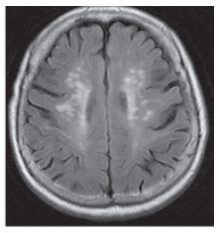

$\mathrm{PVH}=3$
Figure 2. PVH imaging grading.

lyzed collaboratively by two experienced neuroradiologists.

According to Fazekas' rating scale, the extent of PVH was graded as follows: grade 0, absent; grade 1, "caps" or pencilthin lining; grade 2, smooth "halo"; or grade 3, irregular PVH extending into the deep white matter. The extent of DWMH was rated as follows: grade 0 , absent; grade 1 , punctate foci (number of foci $<5$ ); grade 2 (number of foci $>5$ or the diameter of a single foci was $>5 \mathrm{~mm}$ ); or grade 3 , large confluent $\operatorname{areas}^{[15]}$.

\section{Data analysis}

All data obtained in the study were analyzed using SPSS 13.0 for Windows (SPSS, Chicago, IL, USA). The $t$ test was used to compare values of normal distribution in two groups and nonparametric tests were used to analyze the data of non-normal distribution in two groups. Data from the MRI were analyzed by a chi-square test. The grades of DWMH and PVH were combined to yield a score of 1 to 5:1 indicating only slight injury and 5 indicating severe injury. For example, for subjects with " 0 " and " 1 " for DWMH and PVH, respectively, the grades were combined and the injury was considered mild. Similarly, in subjects with grades of " 2 " and " 3 " for DWNH and $\mathrm{PVH}$, respectively, the grades were combined for a total score of 5 , representing severe injury. $P<0.05$ was considered statistically significant between the two groups.

\section{Results}

Initiation years and duration of HRT in subjects

Approximately $66 \%$ of postmenopausal women began their HRT between 50 to 59 years old, and 26\% women had received HRT between 60 to 64 years old (Figure 3). In other words, approximately $91 \%$ of postmenopausal women turned to HRT. Distribution of HRT duration is illustrated in Figure 4. The HRT duration of approximately $49 \%$ of postmenopausal

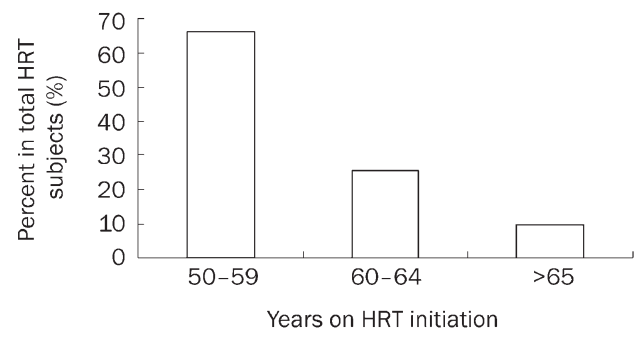

Figure 3. Distribution of the ages of subjects on HRT initiation.

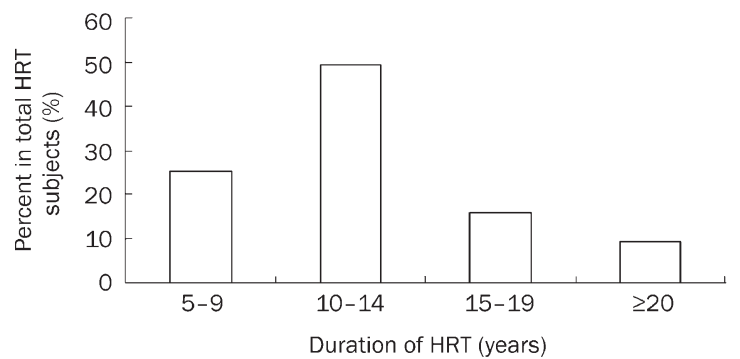

Figure 4. Duration and distribution of HRT subjects.

women was between 10 and 14 years. The duration of treatment in $15 \%$ of the women fall in the range of 15 to 19 years. Taken together, approximately $75 \%$ of women received HRT for more than 10 years.

Plasma level of sex hormones and MMSE evaluation of all age groups

Among the three different age groups, the plasma $\mathrm{E}_{2}$ concentrations in the long-term, low dose HRT groups were maintained above normal levels $(>73.2 \mathrm{pmol} / \mathrm{L})$, whereas those of the control groups were much lower. The difference between the two groups was significant $(P<0.05)$. No significant differences were observed in plasma P or T concentrations, and no differences were observed in the MMSE evaluation between the HRT and controls for all age groups (Table 1).

Blood lipids, glucose, and pressure measurements in all age subgroups

In the 60-69 and 70-87 age subgroups, the cholesterol levels of HRT subjects were lower than those of corresponding control subjects $(5.24 \pm 0.16$ vs $5.96 \pm 0.18, P<0.05$ and $5.56 \pm 2.00$ vs $5.72 \pm 6.72$, respectively). No significant differences were observed in the blood parameters of the HRT and control subgroups (Table 2).

Measurement of DWMH and PVH using MRI in all age subgroups The grading standards for DWMH and PVH are described in the methods section, and representative images are presented in Figures 1 and 2, respectively. In the 50-59 age subgroup, no severe pathological imaging was observed and there was no significant difference in DWMH imaging between the HRT and control group. In the 60-69 age subgroup, 10 cases of severe DWMH were observed in the control group. Although the incidence of severe change was higher in the HRT group than in the controls, there was no statistically significant difference between the two groups. In the 70-87 age subgroup, we observed 31 cases of severe grade changes, which were the most among the three age subgroups. We observed 24 severe grade changes in control group, but only seven in the HRT group. In addition, the grading distribution of DWMH was much higher in the control group than in the HRT group $(P=0.0013)$. No significant differences were observed in PVH between the two groups for all age subgroups (Table 3). 
Table 1. Plasma level of sex hormones and MMSE in different age subgroups. ${ }^{b} P<0.05$ vs corresponding control subgroup.

\begin{tabular}{|c|c|c|c|c|c|}
\hline \multicolumn{6}{|l|}{$50-59$} \\
\hline Control & 19 & $41.09 \pm 13.87$ & $1.088 \pm 0.191$ & $0.775 \pm 0.066$ & $29.63 \pm 3.68$ \\
\hline \multicolumn{6}{|l|}{$60-69$} \\
\hline HRT & 31 & $91.61 \pm 22.16^{b}$ & $1.122 \pm 0.064$ & $0.986 \pm 0.076$ & $29.03 \pm 0.20$ \\
\hline HRT & 21 & $86.41 \pm 17.94^{\mathrm{b}}$ & $1.050 \pm 0.076$ & $0.879 \pm 0.107$ & $28.57 \pm 0.75$ \\
\hline Control & 30 & $45.01 \pm 6.30$ & $1.186 \pm 0.095$ & $0.758 \pm 0.076$ & $28.66 \pm 0.30$ \\
\hline
\end{tabular}

Data from 60-69 age group were analyze by $t$ test and other data were analyzed by non-parametric Wilcoxon rank test.

Table 2. Blood glucose, lipid, and pressure in different age subgroups. ${ }^{b} P<0.05$ vs the corresponding control subgroup.

\begin{tabular}{|c|c|c|c|c|c|}
\hline $\begin{array}{l}\text { Age subgroups } \\
\text { (Years) }\end{array}$ & $\begin{array}{l}\text { Glucose } \\
(\mathrm{mmol} / \mathrm{L})\end{array}$ & $\begin{array}{l}\text { Cholesterol } \\
(\mathrm{mmol} / \mathrm{L})\end{array}$ & $\begin{array}{r}\text { Triglyeride } \\
(\mathrm{mmol} / \mathrm{L})\end{array}$ & $\begin{array}{l}\text { Systolic pressure } \\
\qquad(\mathrm{kPa})\end{array}$ & $\begin{array}{l}\text { Diastolic pressure } \\
\qquad(\mathrm{kPa})\end{array}$ \\
\hline \multicolumn{6}{|l|}{$50-59$} \\
\hline HRT & $5.59 \pm 0.17$ & $5.01 \pm 0.24$ & $1.25 \pm 0.18$ & $16.13 \pm 0.45$ & $10.65 \pm 0.43$ \\
\hline Control & $5.75 \pm 0.37$ & $4.99 \pm 0.67$ & $1.46 \pm 0.23$ & $15.72 \pm 0.50$ & $10.12 \pm 0.31$ \\
\hline \multicolumn{6}{|l|}{$60-69$} \\
\hline \multicolumn{6}{|l|}{$70-87$} \\
\hline HRT & $5.43 \pm 3.45$ & $5.56 \pm 2.00^{b}$ & $1.54 \pm 0.12$ & $17.15 \pm 0.45$ & $10.43 \pm 0.19$ \\
\hline Control & $5.47 \pm 0.20$ & $5.72 \pm 6.72$ & $1.66 \pm 0.13$ & $18.49 \pm 0.48$ & $10.75 \pm 0.25$ \\
\hline
\end{tabular}

Data from 60-69 age group were analyze by $t$ test and other data were analyzed by non-parametric Wilcoxon rank test.

Table 3. Effects of HRT on DWMH and PVH of brain imaging among different age subgroups. ${ }^{\mathrm{b}} \mathrm{P}<0.05$ vs the corresponding control subgroup.

\begin{tabular}{|c|c|c|c|c|c|c|c|c|}
\hline \multirow[t]{2}{*}{$\begin{array}{c}\text { Age subgroups } \\
\text { (Years) }\end{array}$} & \multicolumn{3}{|c|}{$\begin{array}{l}\text { Number and percent of subjects in } \\
\text { different DWMH grading levels }\end{array}$} & \multicolumn{5}{|c|}{$\begin{array}{l}\text { Number and percent of subjects in } \\
\text { different PVH grading levels }\end{array}$} \\
\hline & $0-1$ & $2-3$ & Total & $P$ value & $0-1$ & $2-3$ & Total & $P$ value \\
\hline \multicolumn{9}{|l|}{$50-59$} \\
\hline HRT & $13(68.4 \%)$ & $6(31.6 \%)$ & 19 & & 17 (89.5\%) & $2(10.5 \%)$ & 19 & \\
\hline Control & $18(94.7 \%)$ & $1(5.3 \%)$ & 19 & 0.0897 & 19 (100.0\%) & $0(0.0 \%)$ & 19 & 0.2432 \\
\hline Total & 31 (81.6\%) & $7(18.4 \%)$ & 38 & & $36(94.7 \%)$ & $2(5.3 \%)$ & 38 & \\
\hline \multicolumn{9}{|l|}{$60-69$} \\
\hline HRT & 19 (59.4\%) & $13(40.6 \%)$ & 32 & & $24(75.0 \%)$ & $8(25.0 \%)$ & 32 & \\
\hline Control & $24(70.6 \%)$ & $10(29.4 \%)$ & 34 & 0.3393 & $26(76.5 \%)$ & $8(23.5 \%)$ & 34 & 0.8892 \\
\hline Total & $43(65.2 \%)$ & $23(34.8 \%)$ & 66 & & $50(75.8 \%)$ & $16(24.2 \%)$ & 66 & \\
\hline \multicolumn{9}{|l|}{$70-87$} \\
\hline HRT & $13(65.0 \%)$ & 7 (35.0\%) & 20 & & 11 (55.0\%) & $9(45.0 \%)$ & 20 & \\
\hline Control & $6(20.0 \%)$ & $24(80.0 \%)$ & 30 & $0.0013^{b}$ & $11(36.7 \%)$ & $19(63.3 \%)$ & 30 & 0.2008 \\
\hline Total & $19(38.0 \%)$ & $31(62.0 \%)$ & 50 & & $22(44.0 \%)$ & $28(56.0 \%)$ & 50 & \\
\hline
\end{tabular}

All data were analyzed by chi-square test. Definite probability method of fourfold table was employed to calculate the $x^{2}$ value.

\section{Discussion}

The early use of low dose HRT on postmenopausal women was proposed by PUMCH as early as the 1960s. Using HRT helped to avoid the adverse reaction and side effects of menopause and delivered protective effects on postmenopausal osteoporosis and atherosclerosis of the cardiovascular system. 
Our previous study reported that the age-related atrophy of the hippocampus was much slower in the long-term, low dose HRT group than in the controls ${ }^{[10,11]}$. There is, however, some debate regarding the effects of HRT in postmenopausal women. Many studies have reported negative results of HRT or have suggested risks that increase during HRT. Among those clinical trials, the Women's Health Initiative Memory Study project, completed in 2004, failed to find any positive effects of HRT. It is worth noting that the present study is a retrospective, cross-sectional study, in which the subjects were members of the medical staff at PUMCH. This ensured compliance and reliability in the treatment regimen. As shown in Figures 3 and 4, approximately 75\% of women received HRT for more than 10 years, and about $91 \%$ postmenopausal the women in our study had turned to HRT. There was a significant difference between our study and the Women's Health Initiative Memory Study project, in which the duration of HRT was for 7 years and the initiation of HRT was at 65 years. There was also a difference in the administered dosages. The Women's Health Initiative Memory Study project used the recommended dosage, but the dosage taken by the subjects in our study was less, meeting the concept of "individualized treatment". The results obtained from current study should therefore be taken into consideration when expressing doubt regarding the effects of HRT.

Cerebral anatomy showed that the blood vessels of the white matter around the lateral ventricle were long cortical medullary branches, which lacked vascular anastomosis and resulted in poor blood supply. Furthermore, the blood vessels had to travel long distances $(3-10 \mathrm{~mm})$ to reach the periventricular area, the lumens of which were confined because of long-term hypertension and atherosclerosis in elderly individuals. Thus, the periventricular areas were easily affected by ischemia ${ }^{[16,17]}$.

White matter foci can be detected on an MRI of both healthy elderly people and those that are cognitively impaired. A pathological study of these foci indicated that they were related to types of atherosclerosis, including myelin sheath loss and neuroglia hyperplasia. Some clinical cross-sectional studies abroad have shown gender-related differences in the MRI images of elderly people. The extent and prevalence of white matter harms (WMHs) is much higher in women than in men of the same age ${ }^{[18,19]}$. Another 3-year longitudinal study of 554 elderly people (70 82 years old) had shown that, although the white matter were progressively harmed in both old men and women, the severity in the latter was two-fold higher ${ }^{[20]}$. The authors attributed this difference to increased sensitivity to ischemia in postmenopausal women because of the decline in estrogen levels ${ }^{[20]}$. The longitudinal study that compared the WMH between men and women of the same age better explained the important protective effect of estrogen on silent ischemic brain damage.

We have shown that the plasma levels of estrogen in all HRT subgroups were significantly higher than those of controls $(P<0.05)$, and the grading distribution of DWMH in the HRT group was much lower than that of the control group $(P=0.0013)$ in individuals over 70 . This indicated that HRT had a clear protective effect on brain damage in elderly women, which was related to estrogen levels $(P<0.05)$, but not progesterone levels.

Another study showed differences in WMH between two senile groups. In addition, changes in the cardiovascular system of the participants had shown that, in HRT groups, the plaques of the carotid artery were fewer and smaller, the level of the blood lipids harmful to vessels was much lower and the calcification and extent of stenosis of the coronary artery was mild $^{[21]}$. These findings demonstrate that the protective effect of HRT in the brains of postmenopausal women is correlated with its protective effect of the cardiovascular system.

Neuropsychological scales are currently used to evaluate the therapeutic effect of HRT. In this study, we did not observe any differences between the two groups for all ages. The primary reason for this was that all of the participants were mentally healthy. Additionally, the manifestation of changes in brain function could be slower than the changes at the anatomical level. It is also possible that the high education level of the participants could have concealed the symptoms or that the participants affected were still in the state of subclinical structural brain disease. In conclusion, the results of this study indicate that senile women may benefit from the early initiation of HRT.

\section{Acknowledgements}

Project supported by the Special Foundation from Ministry of Science and Technology of China (1999-045) .

\section{Author contribution}

Qin-sheng GE and Ping-ping ZUO designed research; Yanyong LIU, Ling HU, Chao JI, Dong-wen CHEN, Xi SHEN, Nan YANG, Yun YUE, and Xia HONG performed research; Yanyong LIU and Jing-mei JIANG analyzed data; Yan-yong LIU and Ping-ping ZUO wrote the paper.

\section{References}

1 Shumaker SA, Legault C, Kuller L,Rapp SR,Thal L,Lane DS, Fillit H, et al. Conjugated equine estrogens and incidence of probable dementia and mild cognitive impairment in postmenopausal women: Women's Health Initiative Memory Study. JAMA 2004; 291: 2947-58.

2 Zec RF, Trivedi MA. The effects of estrogen replacement therapy on neuropsychological functioning in postmenopausal women with and without dementia: a critical and theoretical review. Neuropsychol Rev 2002; 12: 65-109.

3 Eberling JL, Wu C, Haan MN, Mungas D, Buonocore M, Jagust WJ. Preliminary evidence that estrogen protects against age-related hippocampal atrophy. Neurobiol Aging 2003; 24: 725-32.

4 Raz N, Rodrigue KM, Kennedy KM, Acker JD. Hormone replacement therapy and age-related brain shrinkage: regional effects. Neuroreport 2004; 15: 2531-4.

5 Erickson KI, Colcombe SJ, Raz N, Korol DL, Scalf P, Webb A. Selective sparing of brain tissue in postmenopausal women receiving hormone replacement therapy. Neurobiol Aging 2005; 26: 1205-13.

6 Raz N, Gunning-Dixon F, Head D, Rodrigue KM, Williamson A, Acker JD. Aging, sexual dimorphism, and hemispheric asymmetry of the cerebral cortex: replicability of regional differences in volume. Neurobiol 
Aging 2004; 25: 377-96.

7 Eberling JL, Wu C, Tong-Turnbeaugh R, Jagust WJ. Estrogen- and tamoxifen-associated effects on brain structure and function. Neuroimage 2004; 21: 364-71.

8 Sullivan EV, Marsh L, Pfefferbaum A. Preservation of hippocampal volume throughout adulthood in healthy men and women. Neurobiol Aging 2005; 26: 1093-8.

9 Eberling JL, Wu C, Haan MN, Mungas D, Buonocore M, Jagust WJ. Preliminary evidence that estrogen protects against age-related hippocampal atrophy. Neurobiol Aging 2003; 24: 725-32.

$10 \mathrm{Mu}$ QW, Xie JX, Weng YQ, Wen ZY. A quantitative MRI study of hippocampal formation, amygdala, temporal horn of lateral ventricle of the normal people from forty years to ninety years of age. Chin J Radio 1998; 32: 817-21.

11 Yue Y, Hu L, Tian QJ, Jiang JM, Dong YL, Jin ZY, et al. Effects of longterm, low dose sex hormone replacement therapy on hippocampusand cognition of postmenopausal women of different apoE genotypes. Acta Pharmacol Sin 2007; 28: 1097-103.

12 Zhang ZX, Hong X, Zhao JH, Huang JB, Wei J, Wang JM, et al. The mini-metal state examination in the Chinese residents populatin aged 55 years and over in the urban and rural areas of Beijing. Chin J Neurol 1999; 32: 149-52.

13 Folstein MF, Folstein SE, McHugh PR. "Mini-Mental State": a practical method for grading the cognitive state of patients for the clinician. J Psychiatr Res 1975; 12: 189-98.
14 Tombaugh TN, Mclntyre NJ: The Mini-Mental State Examination: a comprehensive review. J Am Geriatr Soc 1992; 40: 922-35.

15 Rovaris M, lannucci G, Cercignani M, Sormani MP, De Stefano N, Gerevini S, Comi G, Filippi M. Age-related changes in conventional, magnetization transfer, and diffusion-tensor MR imaging findings: study with wholebrain tissue histogram analysis. Radiology 2003; 227: 731-8.

16 Dubas F, Gray F, Reullet E. Leucoencephalopoathies arteriopathques. Rev Neural 1985; 141: 91-3.

17 De Leeuw FE, Richard F, de Groot JC. Interaction between hypertension, apoE and cerebral white watter lesions. Stroke 2004; 35 : 1057-60.

18 Longstreth WT Jr, Manolio TA, Amold A. Clinical correlates of white matter findings on cranial magnetic resonance imaging of $3301 \mathrm{el}-$ derly people. Stroke 1996; 27: 1274-82.

19 De Leeuw FE, De Groot JC, AchtenE, et al. Prevalence of cerebral white matter lesions in elderly people: a population based magnetic resonance imaging study. J Neurol Neurosurg Psychiatry 2001; 70: 9-14.

20 Van den Heuvel DM, Admiraal-Behloul F, ten Dam VH. Different progression rates for deep white matter hyperintensities in elderly men and women. Neurology, 2004; 63: 1699-701.

21 Ge QS, Tian QJ, Tseng H. Development of low-dose reproductive hormone therapies in China. Gynecol Enducrinol 2006; 22: 1-10. 